\title{
Alternating Copolymerization of Fluoroalkenes with Carbon Monoxide
}

Tomoyuki Fujita, Koji Nakano, Makoto Yamashita and Kyoko Nozaki*

Department of Chemistry and Biotechnology

Graduate School of Engineering

The University of Tokyo

7-3-1 Hongo, Bunkyo-ku, Tokyo 113-8656, Japan

Supporting Information

Contents

DSC charts of poly(1a-alt-CO) S2-3

TG chart of poly(1a-alt-CO) $\quad$ S4

${ }^{31} \mathrm{P}$ and ${ }^{13} \mathrm{C}$ NMR spectra of (dppp)Pd(alkyl)BAr 4 complex $(3 \mathrm{a}(2,1))$

${ }^{31} \mathrm{P}$ NMR and HH COSY spectra of the mixture of (BINAPHOS)Pd(alkyl)BAr 4 


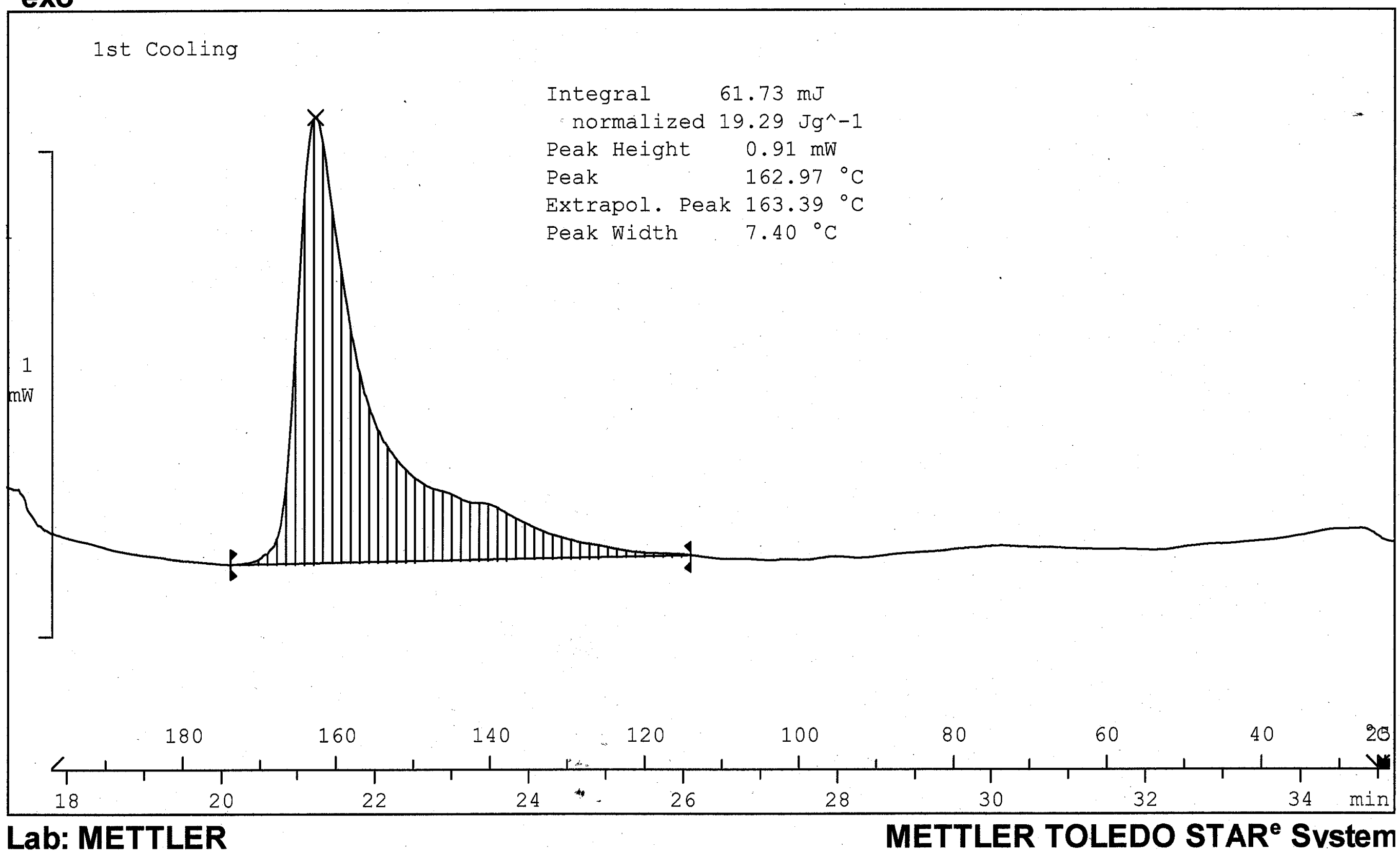




\section{$4 \times 25 a$}

^exo

2nd Heating

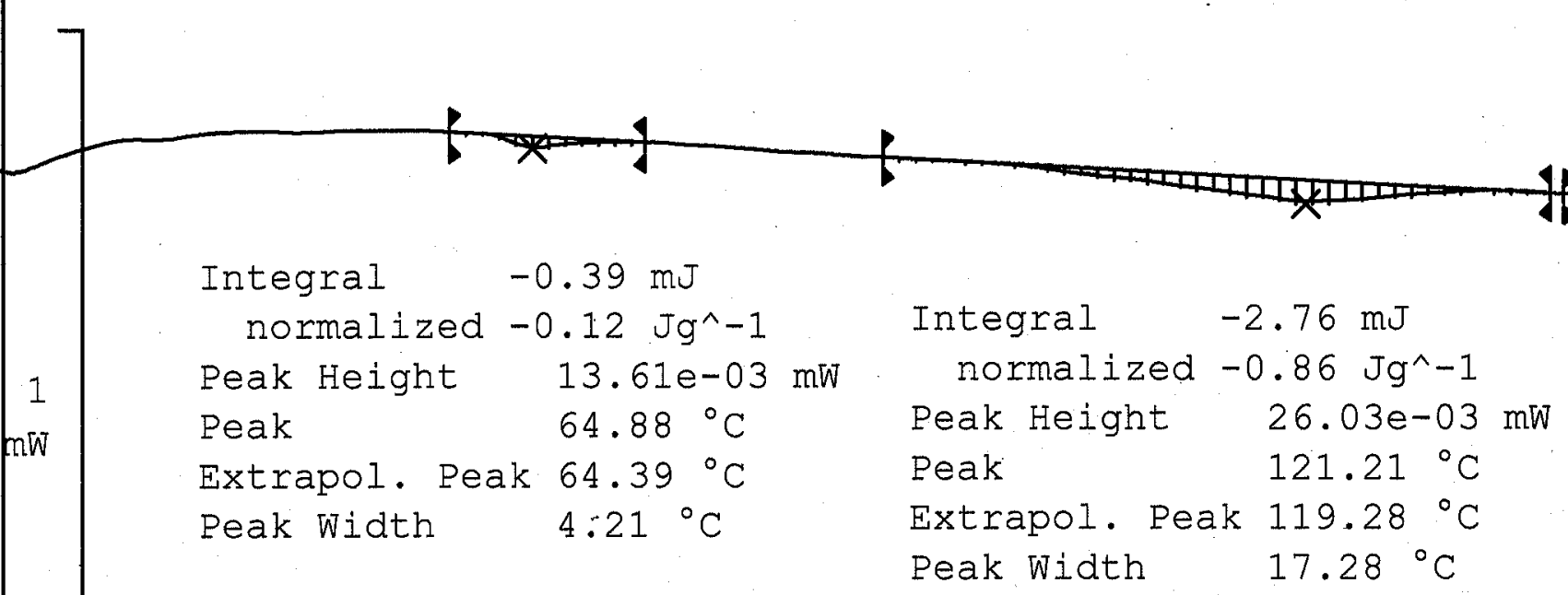

Integral

$-69.45 \mathrm{~mJ}$

normalized $-21.70 \mathrm{Jg}^{\wedge}-1$ ฟ

Peak Height $0.76 \mathrm{mw}$

Peak

$182.64^{\circ} \mathrm{C}$

Extrapol. Peak $183.22{ }^{\circ} \mathrm{C}$

Peak Width $11.88^{\circ} \mathrm{C}$

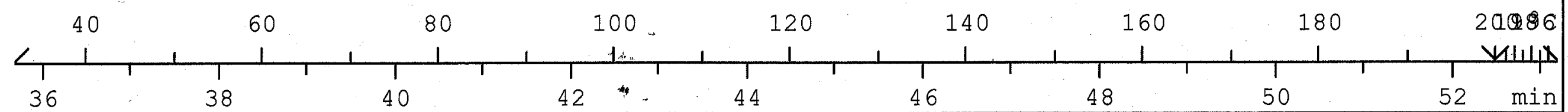




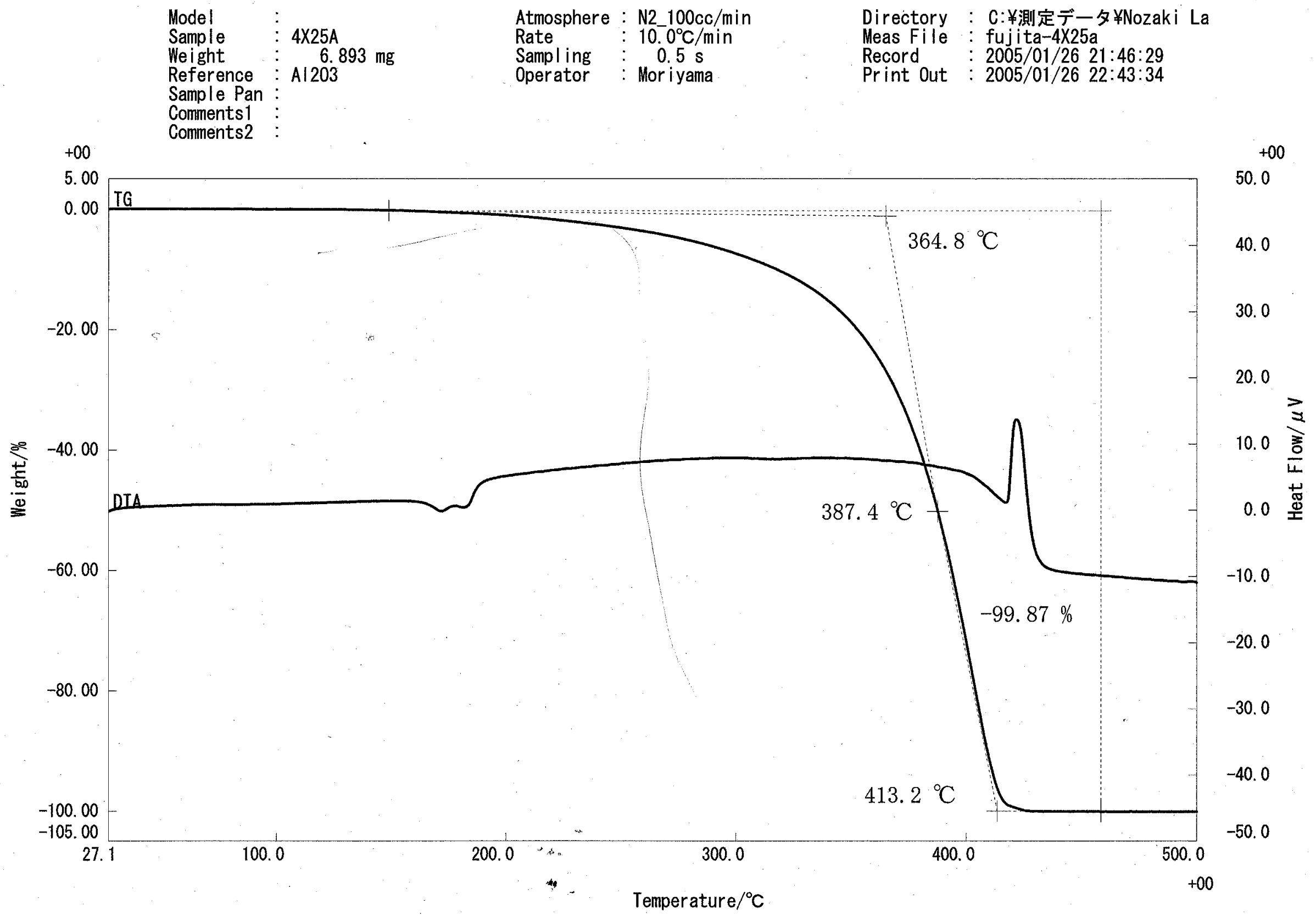


${ }^{31} \mathrm{P}$ NMR spectrum of (dppp)Pd[CH( $\left.\left(\mathrm{CH}_{2} \mathrm{Rf}\right) \mathrm{CH}_{2} \mathrm{COCH}_{3}\right]^{+}\left[\mathrm{BArF}_{4}\right]^{-} \quad[3 \mathbf{a}(2,1)]$

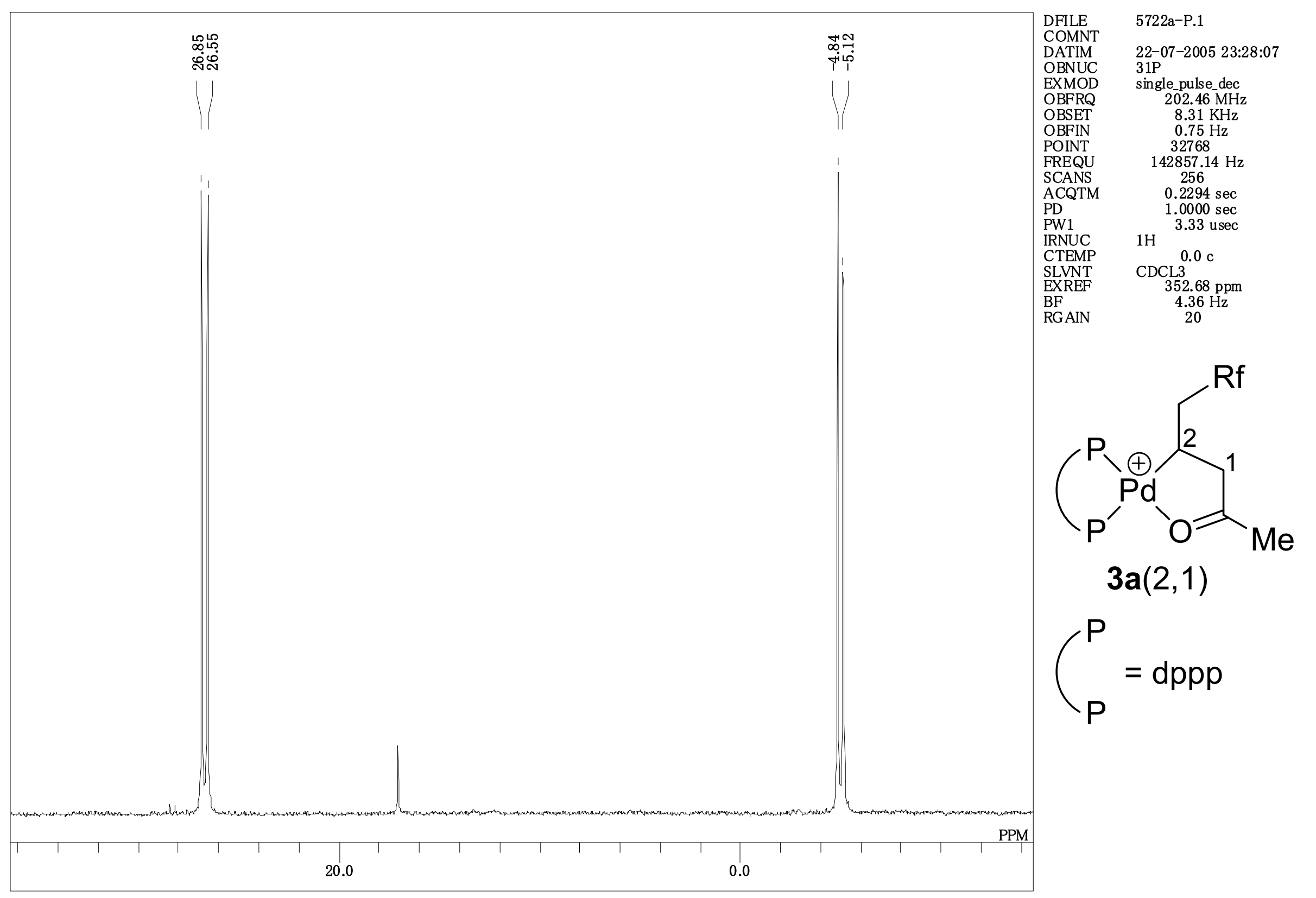


${ }^{13} \mathrm{C}$ NMR spectrum of (dppp)Pd[CH $\left.\left(\mathrm{CH}_{2} \mathrm{Rf}\right) \mathrm{CH}_{2} \mathrm{COCH}_{3}\right]^{+}\left[\mathrm{BArF}_{4}\right]^{-} \quad[3 \mathbf{a}(2,1)]$

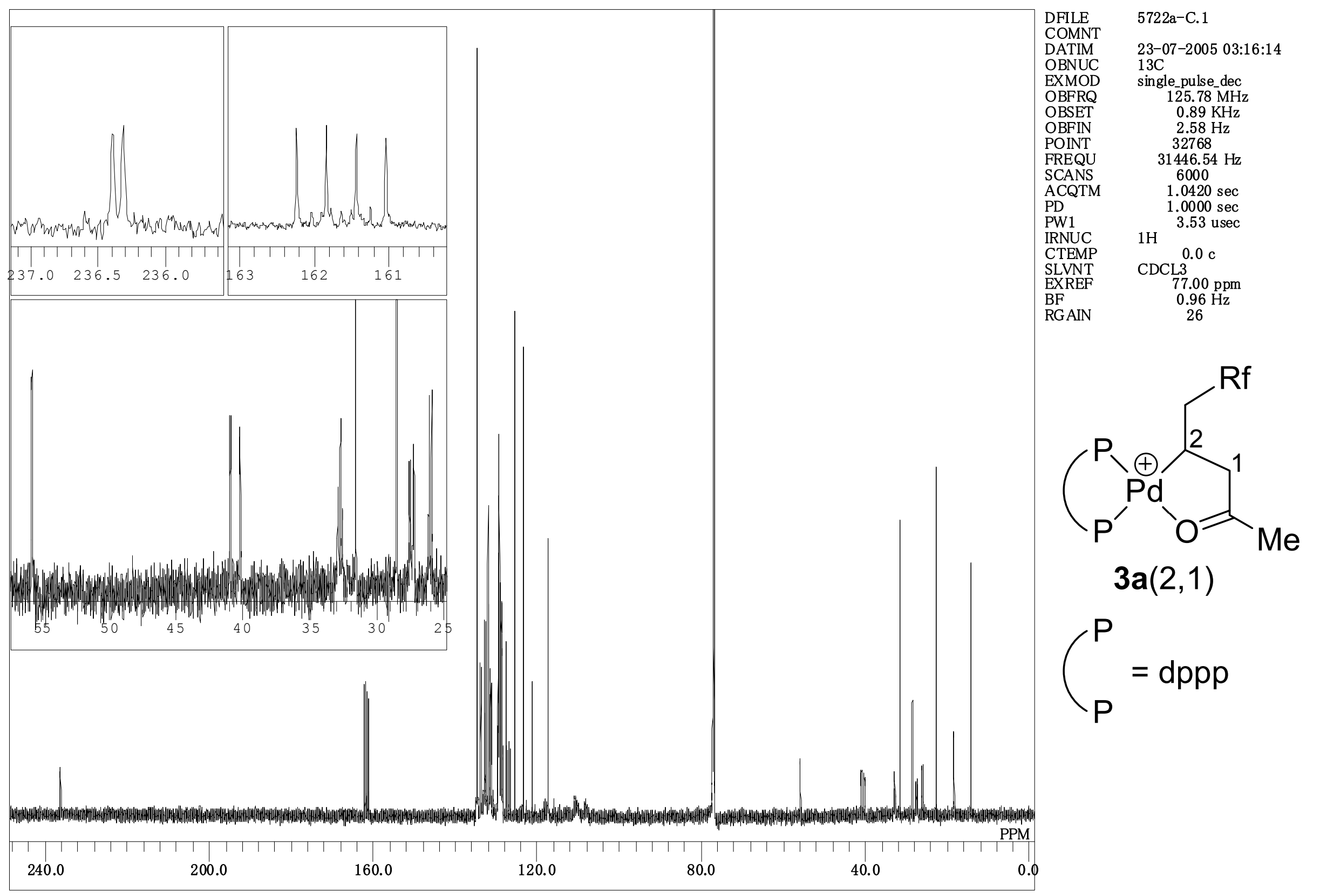


${ }^{31} \mathrm{P}$ NMR spectrum of the isomeric mixture of (BINAPHOS)Pd(alkyl) ${ }^{+}$complexes

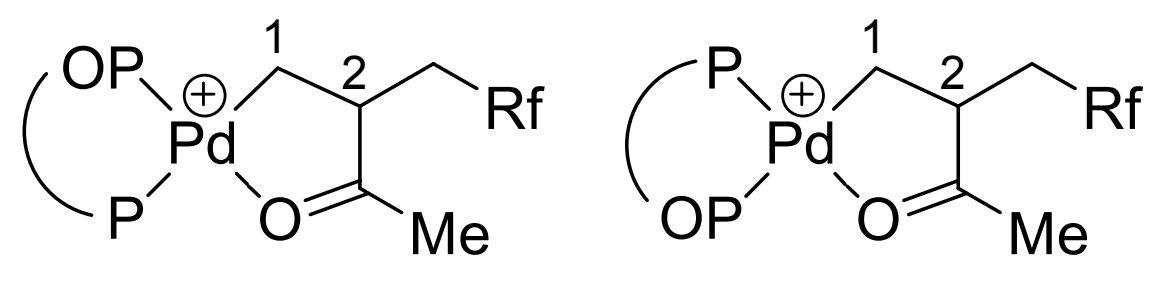

$3 \mathbf{e}(1,2) \mathrm{OP}$-trans-CO $\mathbf{3 e}(1,2) \mathrm{OP}$-cis-CO

${ }^{31} \mathrm{P}$ NMR spectra

$3 \mathbf{e}(1,2)$ OP-trans-CO

$$
3 \mathbf{e}(1,2) \text { OP-trans-CO }
$$

$3 \mathbf{e}(1,2) \mathrm{OP}-$ cis-CO

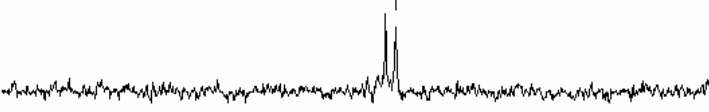

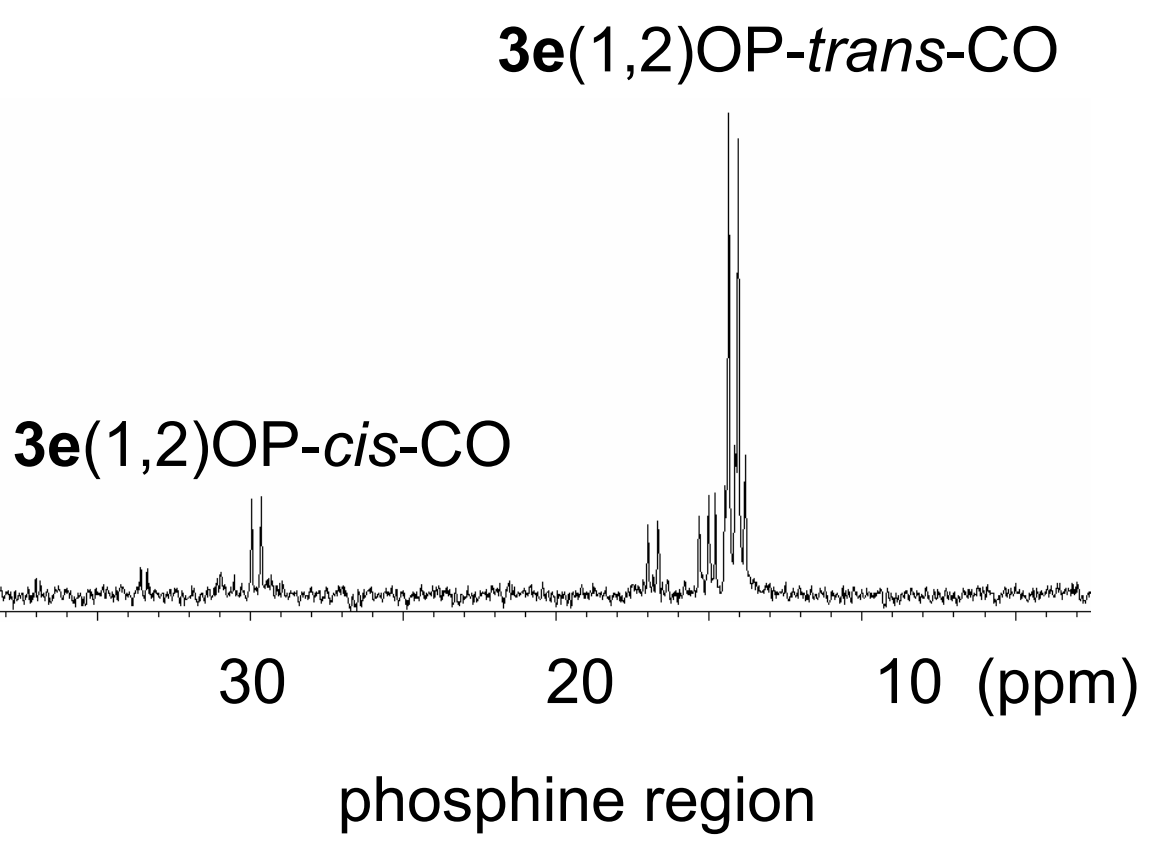

phosphite region

130 (ppm)

150

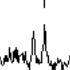

phosphine region 


\section{$\mathrm{HH}$ COSY NMR spectrum of the isomeric mixture of (BINAPHOS)Pd(alkyl) ${ }^{+}$complexes}

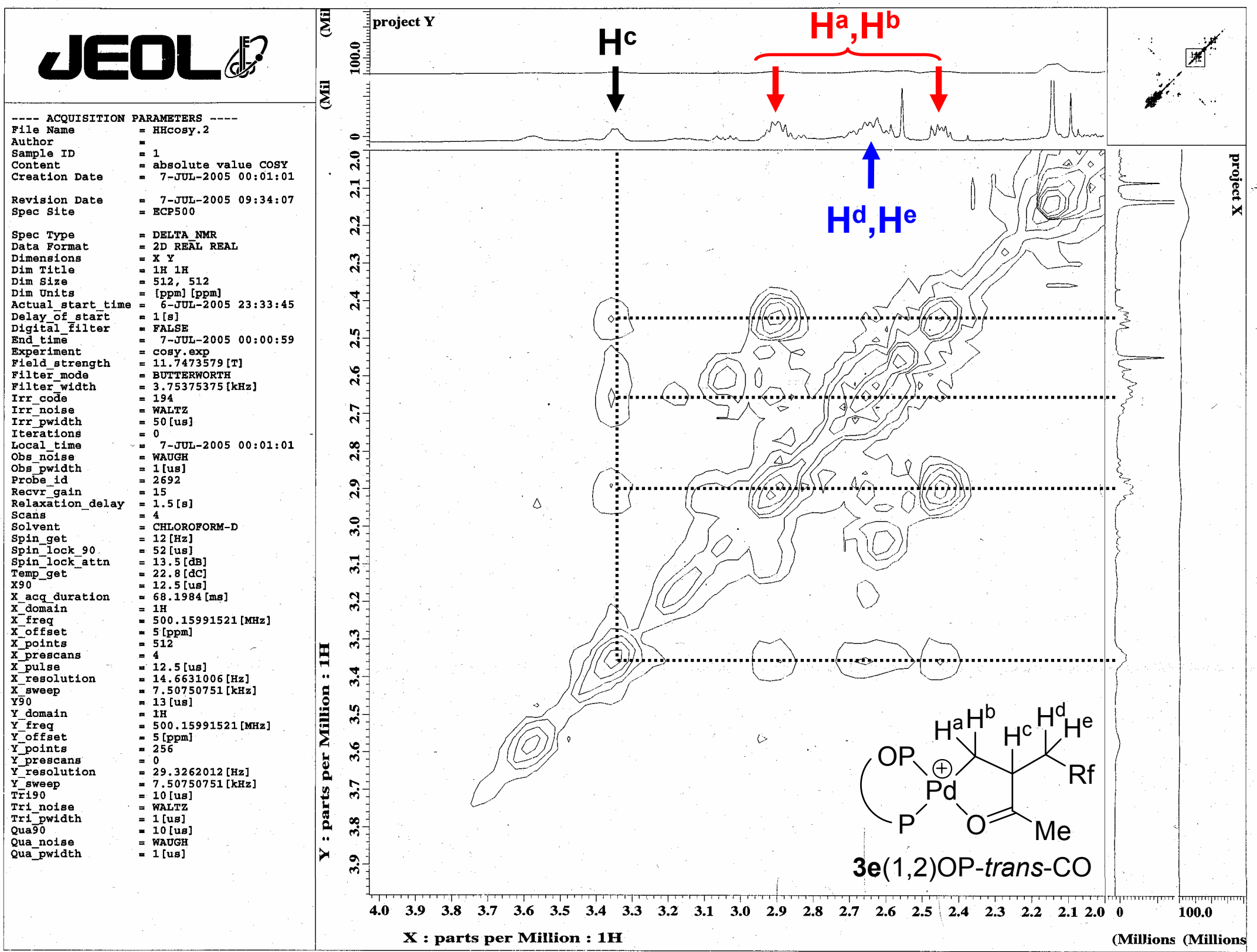

